\title{
STRUCTURE, SURFACE MORPHOLOGY AND OPTICAL PROPERTIES OF THIN FILMS OF ZnS AND CdS GROWN BY ATOMIC LAYER EPITAXY
}

\author{
A. Szczerbakow ${ }^{a *}$, M. Godlewski ${ }^{a}$, E. Dynowska ${ }^{a}$, V.Yu. Ivanov ${ }^{a}$, \\ K. ŚWIĄTEK ${ }^{a}$, E.M. Goldys ${ }^{b}$ AND M.R. PhILliPS ${ }^{c}$ \\ ${ }^{a}$ Institute of Physics, Polish Academy of Sciences \\ Al. Lotników 32/46, 02-668 Warsaw, Poland \\ ${ }^{6}$ Semicond. Sci. \& Technol. Lab., Macquarie Univ., North Ryde, 2109 NSW, Australia \\ ${ }^{c}$ Microstructural Analysis Unit, University of Technology, Sydney, Australia
}

In this communication we report successful growth of monocrystalline cubic $\mathrm{ZnS}$ and monocrystalline and polycrystalline cubic and wurtzite films of CdS by atomic layer epitaxy. Structural and optical properties of these films are analysed. $\mathrm{ZnS}$ (and $\mathrm{CdS} / \mathrm{ZnS}$ ) films grown on GaAs substrate are cubic. Atomic layer epitaxy grown films provide several advantages over $\mathrm{ZnS}$ and CdS materials grown by other techniques, especially compared to bulk material, which is grown at higher temperatures. First results for $\mathrm{ZnS} / \mathrm{CdS} / \mathrm{ZnS}$ quantum well structures are also discussed.

PACS numbers: 68.55.Jk, 68.60.-p, 81.15.Gh

A fundamental benefit of the atomic layer epitaxy (ALE) method is that chemical reactions proceed only in the adsorbed film. This allows to avoid the common problem of conventional chemical vapour deposition (CND) processes, where gas precursors also react in the vapour phase above the substrate. Until now wurtzite films of $\mathrm{ZnS}$, used in thin film electroluminescence displays [1], were deposited by the ALE method on either $\mathrm{Al}_{2} \mathrm{O}_{3}$-covered or uncovered soda glass substrates. These films were polycrystalline. Thus, the technique was often called ALE-CVD or atomic layer deposition and correctness of epitaxy-related name was argued. We report here the first successful growth of monocrystalline cubic $\mathrm{ZnS}$ and monocrystalline and polycrystalline films of $\mathrm{CdS}$, and their quantum well (QW) structures, by ALE using the exchange reaction of hydrogen sulphide with chlorides of zinc (for ZnS growth) and cadmium (for CdS growth). Gas-flow version of the ALE, as used by us ${ }^{\dagger}$, offers good possibilities to engage chemical exchange reactions, which, for example, is difficult in the case of ALE processing by molecular beam epitaxy.

${ }^{*}$ fax: 48-22-8430926, e-mail: szczer@ifpan.edu.pl

1 ALE reactor was bought using SEZAM grant of Foundation for Polish Science. 
The ZnS films were deposited on either (111) or (001) GaAs substrates at $510^{\circ} \mathrm{C}$ temperature [2, 3]. Crystal structure of these films and the morphology of their surfaces were studied with X-ray diffraction, reflection high-energy electron diffraction (RHEED) and scanning electron (SEM) and atomic force (AFM) microscopies. The X-ray data of our ZnS films deposited on (001) and (111) GaAs substrates, supplemented by RHEED analysis, show that ZnS films are monocrystalline and cubic. While $\mathrm{ZnS}$ films on (001) GaAs are expected to be cubic (for example by analogy to cubic films of GaN grown on (001) GaAs [4]), pure cubic growth of $\mathrm{ZnS}$ on (111)-oriented GaAs is a new interesting property of the ALE-grown films. Moreover, RHEED investigations indicate that microtwins (inclined planar defects) are absent, even though the ALE-grown films show granular microstructure, as follows from the roughness examination by AFM. The ALE films have relatively flat surfaces, showing only small granular substructure in sub- $\mu \mathrm{m}$ range.

CdS deposition on uncoated and ZnS-coated (001) GaAs was led using $\mathrm{H}_{2} \mathrm{~S}$ and $\mathrm{CdCl}_{2}$ (at the temperature of $460^{\circ} \mathrm{C}$ ) precursors. The substrate temperature was $510^{\circ} \mathrm{C}$, the same as for $\mathrm{ZnS}[2,3]$, and the parameters of $\mathrm{ZnS}$ deposition in the multilayer processing were the same, as used previously. The cubic $\mathrm{CdS}$ films deposited on (001) GaAs substrate coated with ALE-grown ZnS maintain (001) orientation (Fig. 1a). The cubic structure is, however, slightly tetragonally

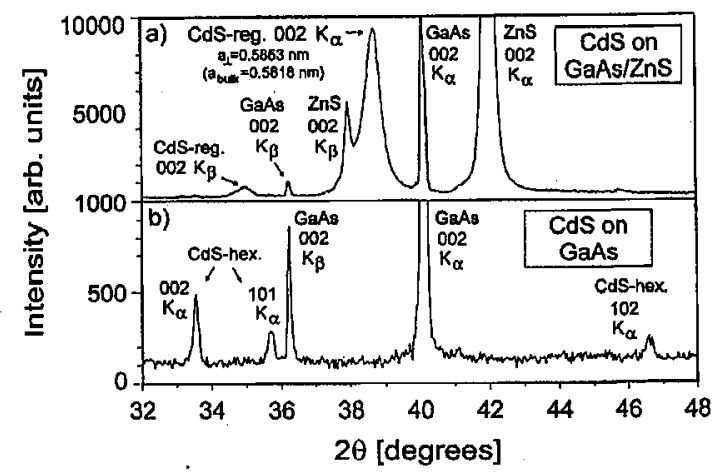

Fig. 1. X-ray diffraction patterns obtained in the "polycrystalline" mode by $\Theta-2 \Theta$ scan with $\mathrm{Co} K_{\alpha}$ radiation, wide slits and sample rotated around normal to the surface of two CdS films grown by ALE on (001) GaAs: covered with $900 \mathrm{~nm} \mathrm{ZnS} \mathrm{layer} \mathrm{(a)} \mathrm{or}$ uncovęred (b).

distorted by strain built into the epilayer. Till now, CdS films grown directly on GaAs substrate (both for (001) and (111) orientations) have been wurtzite and polycrystalline (Fig. 1b). We have also grown first single quantum well (QW) GaAs/ZnS/CdS/ZnS structures on either (001) or (111) GaAs substrates.

Photoluminescence (PL), time-resolved PL and monochromatic, spatially-resolved cathodoluminescence spectroscopy was used to determine optical properties of $\mathrm{ZnS}$ and $\mathrm{CdS}$ films and their QW structures. The relationship between surface morphology and the optical properties of the films was also studied. Both $\mathrm{ZnS}$ and 
CdS films show rather weak self-activated PL emissions. These emissions are weak even though our films are likely Cl-doped, since zinc and cadmium chlorides are used in the growth process. We infer that self-compensation of the $\mathrm{ZnS}$ and $\mathrm{CdS}$ can thus be avoided in the ALE process. This result means that the use of the low growth temperature ALE technique leads to the reduced formation of both planar and intrinsic point defects. This appears to be a very important advantage of the ALE-grown films. For example, very effective self-compensation process leads to a high resistivity material and prohibits wider use of $\mathrm{ZnS}$ material in light emitting devices, for $\mathrm{ZnS}$ grown in a conventional way.

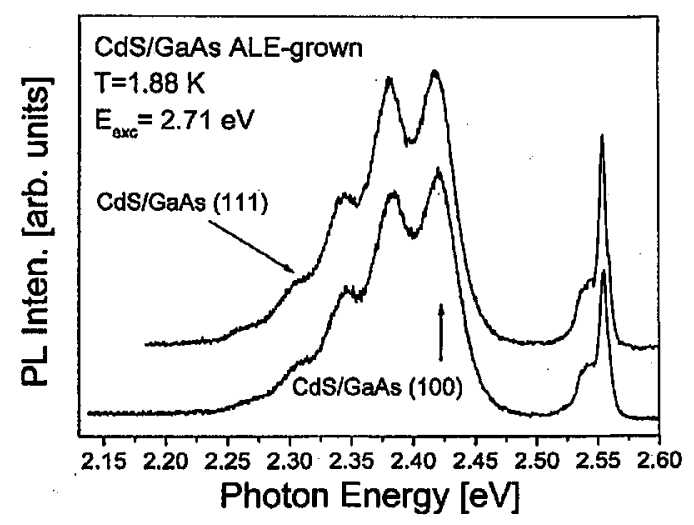

Fig. 2. $2 \mathrm{~K}$ photoluminescence spectrum of two wurtzite CdS epilayers grown on either (001) or (111) GaAs.

In CdS the "edge" PL emission, shown in Fig. 2 for two layers grown directly on $\mathrm{GaAs}$, is dominated by two overlapping transitions of free electron-to-shallow acceptor and shallow donor-to-shallow acceptor pair (DAP) nature, with well-resolved longitudinal optical (LO) phonon replica at each $38 \mathrm{meV}$, which agrees with $38.07 \mathrm{meV}$ value of LO-phonon of bulk CdS crystals of wurtzite structure. The relative intensity of these two emissions depends on excitation conditions and on sample temperature. At low temperature the DAP transition dominates, as confirmed in our time-resolved experiments. We have observed a typical energy-dependent and nonexponential decay of the PL, which is related to the distance distribution of recombining D-A pairs. Unexpectedly, the shallow DAP PL is more intense and is better resolved in polycrystalline wurtzite films than in monocrystalline cubic affected, however, by the lattice parameter mismatch. A double line PL is also observed (see Fig. 2), with the maximum at about $2.555 \mathrm{eV}$. By analogy to bulk CdS, this emission may be due to either A-exciton (observed at $2.552 \mathrm{eV}$ ) or $\mathrm{I}_{2}$-exciton (donor bound exciton).

Shallow DAP PL is also observed in our first $\mathrm{ZnS} / \mathrm{CdS} / \mathrm{ZnS}$ single QW structures grown on either (001) or (111) GaAs. A weaker double line excitonic PL is also observed, with the maxima at about $2.564 \mathrm{eV}$ and $2.547 \mathrm{eV}$ for $\mathrm{QW} 8.5 \mathrm{~nm}$ wide. Confinement energy remains, however, unknown since QW is likely of cubic structure and the band gap energy of cubic CdS remains unknown. It is close to 
that of wurtzite CdS, but exact value is missing [5]. It is difficult to determine this value, at present. We do not know the magnitude of strain effects. For example, the DAP PL has zero-phonon line at about $2.42 \mathrm{eV}$, which is higher by about $25 \mathrm{meV}$ with respect to the relevant position of the shallow DAP recombination in bulk CdS of wurtzite structure. For bulk CdS samples, the shallow DAP transition is related to the $18.7 \mathrm{meV}$ deep donor of unknown chemical origin. In the case of our Cl-rich growth conditions chlorine may be introduced into CdS. Chlorine is likely to introduce shallow donor states in CdS lattice and thus may be active in DAP transitions. We are unable to determine, however, if the shallow donor active in the DAP transition in our samples is of the same origin as for bulk CdS crystals.

In conclusion, we report here successful growth of monocrystalline $\mathrm{ZnS}$ and monocrystalline and polycrystalline CdS films by the ALE method. The ZnS films show good structural quality and are of pure cubic phase. No microtwins are observed and self-compensation mechanism is less efficient. CdS films grown on GaAs coated with $\mathrm{ZnS}$ are cubic and monocrystalline. The films grown directly on GaAs are polycrystalline but show intense PL in the band edge region.

\section{Acknowledgments}

This work was supported by grant No. 2 P03B 8611 of the Committee for Scientific Research (Poland). We are indebted to Dr. P. Brown from Cambridge. University for performing RHEED analysis.

\section{References}

[1] M. Godlewski, M. Leskelä, CRC Crit. Rev. Solid State Mater. Sci. 19, 199 (1994).

[2] A. Szczerbakow, E. Dynowska, M. Godlewski, V.Yu. Ivanov, K. Świattek, in: The Fourth Baltic Symposium on Atomic Layer Epitaxy, Tartu (Estonia) 1997, Ed. A. Rosental, Institute of Physics, Tartu 1997, p. 20.

[3] A. Szczerbakow, E. Dynowska, M. Godlewski, K. Świątek, J. Cryst. Growth 183, 708 (1998).

[4] T.L. Tansley, E.M. Goldys, M. Godlewski, B. Zhou, H.Y. Zuo, in: GaN and Related Materials, Ed. S. Pearton, Gordon and Breach Pub., Amsterdam 1997, p. 233.

[5] M. Cardona, M. Weinstein, G.A. Wolff, Phys. Rev. 140, A633 (1965). 